\title{
Fluctuating asymmetry as a putative marker of human-induced stress in avian conservation
}

\author{
LUC LENS and HILDE EGGERMONT
}

\begin{abstract}
Summary
As anthropogenic stress increasingly affects the viability of natural populations of animals and plants, conservation ecologists are challenged to identify vulnerable populations before their demographic and/or genetic properties become irreversibly affected. Since traditional biomarkers of anthropogenic stress are often cumbersome to measure, and populations may thus go extinct before appropriate data can be obtained, there is a growing interest in individual-based markers that do not require repeated captures, are relatively easy to measure, and allow mitigating action one step ahead. One such marker, left-right asymmetry in bilateral symmetrical traits ("fluctuating asymmetry", FA) has become an established bioassay of the quality and health of individuals and populations in evolutionary-ecological studies. However, the lack of a theoretical framework that predicts under which ecological conditions relationships between FA, stress and fitness can be expected, continues to hamper the use of FA in applied conservation. Here, we briefly review the concept, measurement and analysis of FA, and appraise its expediency in a selection of 21 avian studies covering environmental or genetic stress commonly encountered in conservation biology. The majority of studies met the basic statistical requirements of FA analysis, and two-thirds reported significant, positive relationships with environmental or genetic stress, although with substantial variation among traits, stresses, ages and sexes. In most cases, the observed heterogeneity in relationships with FA could be explained by taking into account both methodological and conceptual issues. Effect sizes ranged from very small (0.02) to very large (0.76), with a weighted average of 0.30 , indicating that on average $9 \%$ of variance in the variable of interest was explained by FA. Given the intrinsic difficulties associated with FA analysis, conservation ecologists are advised to combine information from FA with that of other individual-based biomarkers, such as the study of growth-bar dimensions on developing feathers.
\end{abstract}

\section{Introduction}

Anthropogenic factors increasingly stress natural populations of plants and animals in various and intricate ways, and bioassays are urgently needed that can detect such effects before populations become irreversibly affected. In recent decades, ecologists have studied a wide range of indicators to assess the level of anthropogenic changes on populations. Measures include biotic and abiotic properties of habitats (size, shape, structure, composition, connectivity) and communities (components of species diversity, presence of keystone, umbrella or indicator species, complexity of trophic interactions) (Bergman et al. 2004, Chave and Norden 2007, Diekotter et al. 2007, Fischer and Lindenmayer 2007, Hendrickx et al. 2007 and references therein) and single-species traits (density, sex- or age-structure, population growth rates: Holmes et al. 1996, Burke and Nol 2001, Habib et al. 2007). While often instructive and biologically relevant, such indicators commonly share conceptual and practical problems. First, it is generally unknown which biotic and/or abiotic components (resource limitation, 
environmental impact of pollutants or predators, genetic effects, etc.) most strongly affect a species or community, and to what degree these factors may interact. Second, the most appropriate spatial scale at which to measure environmental variables (core activity area, home range, study plot, habitat fragment, landscape) may be hard to predict, yet strongly influences the strength or direction of biological relationships (Lens et al. 2002c, Willis and Whittaker 2002). Third, environmental variables may show daily, seasonal or yearly fluctuations, or the level of sensitivity of species may vary by age. Hence, knowledge of appropriate temporal scales of measurement is also a basic requirement.

One way to overcome such problems is to study individual-based markers as a proxy for broader-scale biological phenomena. However, the study of traditional endpoints such as survival and reproduction is generally cumbersome, time-consuming and expensive, and may therefore be of limited use when populations face the risk of going extinct. A second-best option is to study phenotypic or physiological proxies of fitness, such as body size, body mass, fat-free mass, quality or quantity of hormones (or other components of blood or urine), or the rate or precision of growth processes. As the sensitivity of these proxies to particular types of anthropogenic effects is generally unknown (or can only be assessed post hoc), candidate markers should preferably be applicable to diverse biological systems and organisms (Huggett et al. 1992). To allow proper conservation action, they should also not involve lethal sampling, exhaustive training or expensive equipment, and stress-mediated changes should be detectable before direct components of fitness are compromised (see Leung et al. 2001 for a more exhaustive list of criteria to evaluate biomarkers). Fluctuating asymmetry (FA, small random deviations from left-right symmetry in bilateral traits: Ludwig 1932) is believed to fulfill most of the above criteria, based on its presumed ease, low cost, and non-lethality of measurement, its broad application range (a wide variety of organisms show bilaterally symmetrical traits) and link to fundamental life-history components that are more laborious to measure (Møller 1997), its higher sensitivity to stress effects than more direct components of fitness (i.e. survival, Clarke and McKenzie 1992), and its ability to account for synergistic interactions between stresses, and hence to provide a more accurate picture of overall organism quality (Mayer et al. 1992, Clarke 1993).

In recent decades, a large number of FA studies have been conducted on vertebrates, invertebrates and plants (see Møller and Swaddle 1997 for reviews). Some (but not all) of these studies reported increased FA levels when individuals were exposed to exogenous (environmental) stress (Parsons 1990, 1992, Polak and Trivers 1994, Møller and Swaddle 1997) or suffered from endogenous (genetic) stress due to inbreeding (Soulé 1979, Vrijenhoek and Lerman 1982, Leary et al. 1983, 1984, 1985, Clarke et al. 1992, Møller and Swaddle 1997, Lens et al. 2000) or disruption of coadapted gene complexes (Graham 1992, Alibert and Auffray 2003, Erbout et al. in press). Following the publication of two influential papers by Leary and Allendorf (1989) and Clarke (1995), FA has also been promoted as a potential biomarker in conservation biology. Two studies on birds illustrate this case particularly well. In a study on Barn Swallows Hirundo rustica, Møller (1993) showed a significant increase in male tail feather FA in birds captured in Chernobyl after the 1986 nuclear accident compared to pre-1986 museum samples from the same site and concurrent samples from an uncontaminated site. More asymmetric males bred later than symmetric ones, whereas no phenotypic changes were measured in females, nor in three other morphological variables in both sexes. In a study of seven Afrotropical bird species, Lens et al. (1999) showed a four- to sevenfold increase in tarsus FA in relation to forest degradation, also in comparison with museum specimens collected 50 years before. In addition, tarsus FA proved to be a more sensitive marker of environmental stress than survival (Lens et al. 2002b), hence supporting the notion that FA may predict future stressmediated changes in fitness before populations become irreversibly affected (early warning system sensu Clarke 1995).

Yet, despite these and other cases whereby FA was clearly informative in a conservation context, the utility of FA as a biomarker of anthropogenic stress remains the subject of vivid 
debate. First, there is no consensus whether other types of bilateral asymmetry (directional asymmetry and anti-symmetry) also reflect the homeostatic ability of individuals (Lens and Van Dongen 2000). Second, relationships of FA with environmental stress, inbreeding and various components of fitness are often organism-, trait- or stress-dependent (e.g. Lens et al. 2002a) and generally weak (Leung and Forbes 1996). Third, despite substantial improvements in the statistical treatment of asymmetry data, the underlying mechanisms of developmental homeostasis remain poorly known (Van Dongen 2007). Fourth, as is the case with all scientific phenomena, adequate appraisal of FA may further be hampered by selective reporting, i.e. the reduced likelihood of reporting non-significant research findings (sensu Begg 1994). Selective reporting is believed to conform with the notion of a scientific "revolution" (Kuhn 1970), in which the early phase of a new field of research is characterized by a publication bias toward positive results, a less critical approach to research, or both (Simmons et al. 1999). However, based on visual inspection of funnel graphs (Light and Pillemer 1984; but see Terrin et al. 2005 for a critical view on this tool), Lens et al. (2001) did not find evidence for selective reporting in a meta-analysis of relationships between FA and stress.

In view of the continued interest in FA as a putative marker of human-induced stress in avian conservation, and the accumulating body of literature on this topic, we here provide a concise overview of the concept, measurement and technicalities associated with the analysis of FA data. Next, we present a list of 21 non-experimental studies on tropical and temperate bird species in which FA has been used as a marker of anthropogenic stress. Rather than exploring general relationships by pooling effect sizes in a formal meta-analysis, we aim to assess the usefulness of FA in individual case studies, i.e. under logistic and time constraints usually encountered in the field of applied conservation.

\section{Concept and analysis of fluctuating asymmetry (FA)}

\section{$F A$ as a measure of developmental stability}

Left and right sides of bilateral traits, such as wings, legs or eyes, often show small deviations from perfect symmetrical development. As corresponding body sides share a single genome and tend to experience identical external effects, such deviations cannot be due to genetic or environmental effects per se (Reeve 1960). Rather, the observed asymmetries are believed to reflect the inability of individuals to buffer their development against small, random perturbations ("developmental noise": Palmer 1994; Auffray et al. 1999). Mechanisms underlying such developmental noise are still poorly understood, yet most likely involve perturbations of cellular processes or random variation in physiological rates, both of which may affect rates of cell growth, division, or elongation (Palmer 1994, McAdams and Arkin 1999, Fiering et al. 2000). Innate homeostatic processes buffering the disruptive effects of developmental noise during ontogeny (developmental stability) generally cause a decrease in the magnitude of left-right asymmetries as bilateral traits develop (e.g. Swaddle and Witter 1997, Talloen et al. in press). As a result, non-directional deviations in the development of bilaterally symmetrical traits may be viewed as the outcome of two opposing forces: those tending to disrupt development (developmental noise) and those tending to stabilize it (developmental stability) (Van Valen 1962, Palmer 1994, Klingenberg and Nijhout 1999).

The degree of developmental stability of individuals or populations is most often estimated by their level of FA (Ludwig 1932). FA refers to a pattern of bilateral variation in a sample of individuals, where the mean of right minus left values of a trait is zero and the variation is normally distributed about that mean (Palmer 1994). The appeal of FA as a measure of developmental stability is fourfold. First, FA is one of few morphological attributes for which the norm, i.e. perfect symmetry, is known (Palmer 1996). Second, FA is believed to be a more sensitive marker of stress than traditional fitness measures (e.g. survival: Clarke and McKenzie 1992, Lens et al. 2002b). Third, adequate sample sizes can rather easily be obtained, especially 
when compared to estimates of survival or (lifetime) reproduction success that often require (multiple) recaptures or the fulfilment of stringent model assumptions. Fourth, measurement of FA does not require expensive equipment and can be conducted in a non-destructive manner.

Exogenous or endogenous factors may affect FA by increasing the amount of developmental noise, by decreasing the level of developmental stability, or both (Klingenberg and Nijhout 1999, Klingenberg 2001). Early studies on individual and population levels of FA revealed positive relationships with a wide range of abiotic, biotic and genetic stress effects, yet they also showed variable effects among taxa, traits and types of stress (e.g. Leary and Allendorf 1989, Kieser and Groeneveld 1991, Palmer and Strobeck 1992, Parsons 1990, 1992, Clarke 1993, 1995, Polak and Trivers 1994, Lens et al. 1999, 2000). In a review of 21 experimental tests of relationships between FA and environmental stress (Bjorksten et al. 2000), one-third of the studies showed a consistent increase in FA with stress, one-third showed a trait- or stress-specific increase, and one-third showed no effect. Despite the debate between advocates and critics of the use of FA as a bioassay, attempts to understand the factors that may cause such observed discordance in reported relationships, remain surprisingly scarce.

\section{Measurement and analysis of $F A$}

One of the most attractive properties of FA is the relative ease with which measurements can be taken. Most animal species, and vertebrates in particular, possess multiple bilateral traits that are potentially suitable for the study of FA. In birds, traits comprise tarsi, homologous pairs of primaries, secondaries, tertials and rectrices, and various secondary sexual characteristics (wingbars, eye-rings, supercilia, streamers, wattles, etc.). Apart from external traits, some avian studies have also included skeletal measurements. However, as the latter implies sacrificing individuals (e.g. Karvonen et al. 2003) or the use of expensive equipment (e.g. X-rays: Van Nuffel et al. 2007), such measurements seem less suitable for conservation studies. Unlike many other biomarkers, FA can also be measured on dead animals, such as museum specimens. When comparing museum specimens with live birds, possible effects of (directional) post mortem shrinkage should be taken into account (Bjordal 1983; see Møller 1993, Lens et al. 1999 for valid comparisons).

To be able to separate measurement error (ME) from left-right asymmetry in trait dimensions (number, size, area or shape), at least two measurements or counts at each trait side are required. To maximize the level of statistical independence, repeated measurements are typically taken as sequences of left 1 , right 1 , left 2, right 2, or right 1, left 1, right 2, left 2. Depending on the level of measurement accuracy, a higher number of repeats per trait side may be required in order to maximize the probability of detecting left-right asymmetries (Van Dongen 1999, Van Poucke et al. in press). Prior to the collection of extensive datasets, it is therefore recommended to estimate the proportion of variation in trait size due to ME (i.e. between repeated measurements within sides) and FA (i.e. between sides) (see Van Dongen 1999 for a discussion of effects of sample size on accuracy and power in FA studies).

While some studies suggest that FA, directional asymmetry (DA; normal distribution with non-zero mean) and anti-symmetry (AS; bimodal distribution with zero mean) form part of a continuum and may all represent the ability of an individual to develop along a stable pathway (Graham et al. 1993, Lens and Van Dongen 2000, Kark et al. 2001), DA and AS are generally considered inappropriate for the estimation of developmental stability, owing to their presumed heritable component (Palmer and Strobeck 1992, Palmer et al. 1994; but see Leamy 1999 for an example of low heritability of DA). When estimating individual or population levels of FA, therefore, it is necessary to test for DA and AS and, if present, to correct for it. Statistical analysis of FA hence typically comprises four consecutive steps: (i) to estimate the variance due to $\mathrm{ME}$ and test whether is heterogeneously distributed among samples (e.g. populations, years, treatments); (ii) to separate ME from FA and test for DA and AS; (iii) to verify the statistical significance of FA; and (iv) to compute a single unbiased FA value per individual. 
Various statistical protocols have been developed to perform these tests. The mixed regression model with restricted maximum likelihood (REML) parameter estimation, developed by Van Dongen et al. (1999), is both comprehensive and user-friendly as it can be performed with standard statistical software. The outcome of the analysis is a list of individual FA estimates that constitute the raw data for hypothesis-testing at individual level (regressing FA with estimates of health, survival or reproductive success) or at population level (comparing FA between populations, treatments or years). To examine which traits are most suitable for hypothesistesting, Van Nuffel et al. (2007) developed a selection protocol based on the statistical significance of FA, the absence of other types of bilateral asymmetry, the absence of betweentrait correlation in signed FA values, and the level of signal(FA)-to-noise(ME) ratio. After final selection of the appropriate traits, multiple-trait estimates can be analysed with the use of composite indices of FA, through multivariate analysis, or by structural equation modelling (see Lens et al. 2002a).

\section{FA as a marker of stress in avian conservation}

To appraise the value of FA as a bioassay of human-induced stress in avian conservation, we searched the electronic databases Current Contents ${ }^{\circledR}$ and Web of Science ${ }^{\circledR}$ by submitting different combinations of the keywords "fluctuating asymmetry", "bilateral asymmetry", "developmental stability" and "birds". This produced a list of 151 case studies, all of which we managed to retrieve. Subsequently, we excluded functional-morphological studies, studies on domesticated species, and experimental studies. While the latter may provide more powerful evidence than correlational studies, we specifically aimed at assessing the suitability of FA as a marker of stress under natural conditions where relationships are typically confounded by various sources of unexplained variation. We retained studies addressing distributional edge effects, effect of drought, extreme weather events, fire, and parasites, as these may increasingly affect natural populations under global change. After selection, we obtained a subsample of 21 case studies which we believe are representative of conditions frequently encountered in avian conservation biology.

Each selected paper was independently screened by both authors to extract the following information: country of study, habitat type, type of stress and/or life-history trait involved in hypothesis-testing, species, life stage, number of individuals (nests, populations), and bilateral trait type(s). From a statistical perspective, we determined whether hypothesis-testing was conducted at the population level (i.e. averaging values across nests, populations, species, assemblages, etc.) or at the individual level. We further determined whether or not repeated measurements were taken and directional asymmetry and anti-symmetry was examined and, if present, corrected for (both properties serve as a crude indicator of the statistical robustness of FA estimation; see above). Statistics describing the strength, direction or significance of relationships between individual- or population-level estimates of FA and stress (i.e. sample means, $P$-values, $r_{s}$-values, $F$-values, $U$-values, etc.) were translated into a standard measure of effect size (Rosenthal 1991) using the program META-ANALYSIS 5.3 (Schwarzer 1989). Based on developmental theory (see above), we used positive effect sizes $(r)$ to indicate positive relationships between FA and stress. Since effect sizes were merely used for descriptive (rather than analytical) purposes, multiple outcomes of the same study were treated as independent data when calculating average effect sizes, weighted by sample size. After screening each paper, all first authors were contacted to evaluate the accuracy of our assessment and provide additional information, if available.

Case studies comprised temperate as well as tropical species, both passerines and nonpasserines, and were conducted in a variety of habitats ranging from temperate urban areas to tropical rainforests (Table 1). Presumed stress effects were related to degradation or fragmentation of natural habitats (edge effects, isolation, vegetation structure, desiccation, food limitation), environmental pollution (pesticides, heavy metals, radioactivity), infection with 
Table 1. Avian field studies (1993-2007) using FA as bioassay for human-induced stress effects. RM, repeated measurements; DA, test for directional asymmetry; AS, test for anti-symmetry; yes(+), significant result in expected direction; yes (-), significant result but not in expected direction; n.a., not applicable. See text for details on selection criteria.

\begin{tabular}{|c|c|c|c|c|c|c|c|c|c|c|c|c|c|c|}
\hline Reference & Country & Habitat & Stress & Level & Species & Age/Sex & $\begin{array}{l}\text { \# individuals } \\
\text { or nests }\end{array}$ & Bilateral trait & $\begin{array}{l}\text { Individual } \\
\text { covariate }\end{array}$ & RM & $\overline{\mathrm{DA}}$ & $\mathrm{AS}$ & $\begin{array}{l}\text { Significant } \\
\text { effect }\end{array}$ & $\begin{array}{l}\text { Effect } \\
\text { size }\end{array}$ \\
\hline \multirow[t]{12}{*}{$\begin{array}{l}\text { Anciães } \\
\text { and Marini } \\
2000\end{array}$} & Brazil & $\begin{array}{l}\text { semi- } \\
\text { deciduous) } \\
\text { tropical } \\
\text { rainforest }\end{array}$ & $\begin{array}{l}\text { habitat } \\
\text { fragmentation }\end{array}$ & population & $\begin{array}{l}\text { Dysithamnus } \\
\text { mentalis }\end{array}$ & not specified & 28 ind. & $\begin{array}{l}\text { flattened wing } \\
\text { length }\end{array}$ & & yes & yes & yes & no & 0.02 \\
\hline & & & & & & & 30 ind. & $\begin{array}{l}\text { tarsus } \\
\text { length }\end{array}$ & & yes & yes & yes & yes $(+)$ & 0.37 \\
\hline & & & & & $\begin{array}{l}\text { Conopophaga } \\
\text { lineata }\end{array}$ & not specified & 48 ind. & $\begin{array}{l}\text { flattened wing } \\
\text { length }\end{array}$ & & yes & yes & yes & yes $(+)$ & 0.29 \\
\hline & & & & & & & 45 ind. & $\begin{array}{l}\text { tarsus } \\
\text { length }\end{array}$ & & yes & yes & yes & no & 0.07 \\
\hline & & & & & $\begin{array}{l}\text { Platyrinchus } \\
\text { mystaceus }\end{array}$ & not specified & 52 ind. & $\begin{array}{l}\text { flattened wing } \\
\text { length }\end{array}$ & & yes & yes & yes & yes $(+)$ & 0.29 \\
\hline & & & & & & & 53 ind. & $\begin{array}{l}\text { tarsus } \\
\text { length }\end{array}$ & & yes & yes & yes & yes $(+)$ & 0.28 \\
\hline & & & & & $\begin{array}{l}\text { Turdus } \\
\text { albicollis }\end{array}$ & not specified & 82 ind. & $\begin{array}{l}\text { flattened wing } \\
\text { length }\end{array}$ & & yes & yes & yes & no & 0.12 \\
\hline & & & & & & & 82 ind. & $\begin{array}{l}\text { tarsus } \\
\text { length }\end{array}$ & & yes & yes & yes & yes $(+)$ & 0.20 \\
\hline & & & & & $\begin{array}{l}\text { Tachyphonus } \\
\text { coronatus }\end{array}$ & not specified & 62 ind. & $\begin{array}{l}\text { flattened wing } \\
\text { length }\end{array}$ & & yes & yes & yes & no & 0.05 \\
\hline & & & & & & & 52 ind. & & & yes & yes & yes & no & 0.17 \\
\hline & & & & & $\begin{array}{l}\text { Trichothraupis } \\
\text { melanops }\end{array}$ & not specified & 99 ind. & $\begin{array}{l}\text { flattened wing } \\
\text { length }\end{array}$ & & yes & yes & yes & no & 0.07 \\
\hline & & & & & & & 95 ind. & $\begin{array}{l}\text { tarsus } \\
\text { length }\end{array}$ & & yes & yes & yes & yes $(+)$ & 0.23 \\
\hline \multirow[t]{6}{*}{$\begin{array}{l}\text { Bustnes } \\
\text { et al. } 2002\end{array}$} & Norway & $\begin{array}{l}\text { coastal } \\
\text { breeding } \\
\text { colony }\end{array}$ & $\begin{array}{l}\text { organochlorine } \\
\text { (OC) pollution }\end{array}$ & individual & $\begin{array}{l}\text { Larus } \\
\text { hyperboreus }\end{array}$ & adults & 86 ind. & $\begin{array}{l}\text { 3rd } \\
\text { primary } \\
\text { length }\end{array}$ & $\begin{array}{l}\text { PCB-99 } \\
\text { concentration }\end{array}$ & yes & yes & yes & yes $(+)$ & 0.22 \\
\hline & & & & & & & & & $\begin{array}{l}\text { PCB-118 } \\
\text { concentration }\end{array}$ & yes & yes & yes & yes $(+)$ & 0.24 \\
\hline & & & & & & & & & $\begin{array}{l}\text { PCB-138 } \\
\text { concentration }\end{array}$ & yes & yes & yes & no & 0.21 \\
\hline & & & & & & & & & $\begin{array}{l}\text { PCB-153 } \\
\text { concentration }\end{array}$ & yes & yes & yes & no & 0.19 \\
\hline & & & & & & & & & $\begin{array}{l}\text { PCB-170 } \\
\text { concentration }\end{array}$ & yes & yes & yes & no & 0.18 \\
\hline & & & & & & & & & $\begin{array}{l}\text { PCB-18o } \\
\text { concentration }\end{array}$ & yes & yes & yes & no & 0.18 \\
\hline
\end{tabular}


Table 1. Continued.

\begin{tabular}{|c|c|c|c|c|c|c|c|c|c|c|c|c|c|c|}
\hline Reference & Country & Habitat & Stress & Level & Species & Age/Sex & $\begin{array}{l}\text { \# individuals } \\
\text { or nests }\end{array}$ & Bilateral trait & $\begin{array}{l}\text { Individual } \\
\text { covariate }\end{array}$ & RM & DA & AS & $\begin{array}{l}\text { Significant } \\
\text { effect }\end{array}$ & $\begin{array}{l}\text { Effect } \\
\text { size }\end{array}$ \\
\hline & & & & & & & & & $\begin{array}{l}\text { Oxychlordane } \\
\text { concentration }\end{array}$ & & yes & yes & yes $(+)$ & 0.21 \\
\hline & & & & & & & & & & yes & yes & yes & yes $(+)$ & 0.26 \\
\hline & & & & & & & & & $\begin{array}{l}\text { HCB } \\
\text { concentration }\end{array}$ & yes & yes & yes & yes $(+)$ & 0.32 \\
\hline \multirow[t]{5}{*}{$\begin{array}{l}\text { Bustnes } \\
\text { et al. } 2007\end{array}$} & Norway & $\begin{array}{l}\text { coastal } \\
\text { breeding } \\
\text { colony }\end{array}$ & $\begin{array}{l}\text { organochlorine } \\
\text { (OC) pollution }\end{array}$ & individual & $\begin{array}{l}\text { Larus } \\
\text { marinus }\end{array}$ & males $^{1}$ & to ind. & $\begin{array}{l}\text { 3rd primary } \\
\text { length }\end{array}$ & $\begin{array}{l}\text { PCB } \\
\text { concentration }\end{array}$ & yes & yes & yes & yes $(+)$ & 0.63 \\
\hline & & & & & & & & & $\begin{array}{l}\text { HCB } \\
\text { concentration }\end{array}$ & yes & yes & yes & no & $--^{2}$ \\
\hline & & & & & & & & & $\begin{array}{l}\text { Oxychlordane } \\
\text { concentration }\end{array}$ & yes & yes & yes & no & $--^{2}$ \\
\hline & & & & & & & & & $\begin{array}{l}\mathrm{p}, \mathrm{p}^{\prime} \text {-DDE } \\
\text { concentration }\end{array}$ & yes & yes & yes & no & $--^{2}$ \\
\hline & & & & & & & & & $\begin{array}{l}\beta \text {-HCH } \\
\text { concentrarion }\end{array}$ & yes & yes & yes & no & $--^{2}$ \\
\hline \multirow[t]{3}{*}{$\begin{array}{l}\text { Brown } \\
\text { and Brown } \\
1998\end{array}$} & USA & $\begin{array}{l}\text { cliffs, } \\
\text { bridges, } \\
\text { buildings }\end{array}$ & $\begin{array}{l}\text { severe } \\
\text { weather } \\
\text { conditions } \\
\text { (food stress) }\end{array}$ & individual & $\begin{array}{l}\text { Petrochelidon } \\
\text { pyrrhonota }\end{array}$ & $\begin{array}{l}\text { fledglings/ } \\
\text { adults }\end{array}$ & 1090 ind. & $\begin{array}{l}\text { outer } \\
\text { rectrix } \\
\text { length }\end{array}$ & survival & yes & yes & yes & yes $(+)$ & 0.41 \\
\hline & & & & & & & & $\begin{array}{l}\text { unflattened } \\
\text { wing length }\end{array}$ & & yes & yes & yes & yes $(+)$ & 0.69 \\
\hline & & & & & & & & tarsus length & & yes & yes & yes & no & 0.35 \\
\hline \multirow[t]{4}{*}{$\begin{array}{l}\text { Brown } \\
\text { and Brown } \\
\text { 1999 }\end{array}$} & USA & $\begin{array}{l}\text { bridges, } \\
\text { buildings, } \\
\text { highway } \\
\text { culverts }\end{array}$ & $\begin{array}{l}\text { severe } \\
\text { weather } \\
\text { conditions } \\
\text { (food stress) }\end{array}$ & individual & $\begin{array}{l}\text { Hirundo } \\
\text { rustica }\end{array}$ & male & 16 ind. & $\begin{array}{l}\text { unflattened } \\
\text { wing length }\end{array}$ & survival & yes & yes & yes & yes $(+)^{3}$ & 0.54 \\
\hline & & & & & & & & $\begin{array}{l}\text { outer rectrix } \\
\text { length }\end{array}$ & & yes & yes & yes & no & 0.48 \\
\hline & & & & & & female & 29 ind. & $\begin{array}{l}\text { unflattened } \\
\text { wing length }\end{array}$ & & yes & yes & yes & no & 0.31 \\
\hline & & & & & & & & $\begin{array}{l}\text { outer rectrix } \\
\text { length }\end{array}$ & & yes & yes & yes & no & $0.4^{2}$ \\
\hline $\begin{array}{l}\text { Carbonell } \\
\text { and Telleria } \\
1998\end{array}$ & Spain & $\begin{array}{l}\text { mixed } \\
\text { forest along } \\
\text { a drought } \\
\text { gradient }\end{array}$ & drought & population & $\begin{array}{l}\text { Sylvia } \\
\text { atricapilla }\end{array}$ & $\begin{array}{l}\text { fledglings/ } \\
\text { adults }\end{array}$ & 173 ind. & tarsus length & & yes & yes & yes & yes $(+)$ & 0.32 \\
\hline
\end{tabular}


Table 1 . Continued.

\begin{tabular}{|c|c|c|c|c|c|c|c|c|c|c|c|c|c|c|}
\hline Reference & Country & Habitat & Stress & Level & Species & Age/Sex & $\begin{array}{l}\# \text { individuals } \\
\text { or nests }\end{array}$ & Bilateral trait & $\begin{array}{l}\text { Individual } \\
\text { covariate }\end{array}$ & RM & $\overline{D A}$ & AS & $\begin{array}{l}\text { Significant } \\
\text { effect }\end{array}$ & $\begin{array}{l}\text { Effect } \\
\text { size }\end{array}$ \\
\hline \multirow[t]{3}{*}{$\begin{array}{l}\text { Carbonell } \\
\text { and Telleria } \\
\text { 1999 }\end{array}$} & \multirow[t]{3}{*}{ Spain } & \multirow[t]{3}{*}{$\begin{array}{l}\text { mixed } \\
\text { forest along } \\
\text { a drought } \\
\text { gradient }\end{array}$} & \multirow[t]{3}{*}{ drought } & \multirow[t]{3}{*}{ population } & \multirow[t]{3}{*}{$\begin{array}{l}\text { Sylvia } \\
\text { atricapilla }\end{array}$} & \multirow[t]{3}{*}{$\begin{array}{l}\text { fledglings/ } \\
\text { adults }\end{array}$} & 95 ind. & rectrix length & & yes & yes & yes & no & 0.03 \\
\hline & & & & & & & 98 ind. & rectric mass & & no & yes & yes & no & 0.18 \\
\hline & & & & & & & 95 ind & $\begin{array}{l}\text { rectrix mass/ } \\
\text { length }\end{array}$ & & na & na & na & yes $(+)$ & 0.28 \\
\hline $\begin{array}{l}\text { Carbonell } \\
\text { et al. } 2003\end{array}$ & Spain & $\begin{array}{l}\text { gradient } \\
\text { from moist } \\
\text { mixed } \\
\text { forest to }\end{array}$ & drought & population & $\begin{array}{l}\text { Sylvia } \\
\text { atricapilla }\end{array}$ & $\begin{array}{l}\text { fledglings/ } \\
\text { adults }\end{array}$ & 97 ind & $\begin{array}{l}\text { tarsus } \\
\text { length }\end{array}$ & & yes & yes & yes & yes $(+)$ & 0.22 \\
\hline \multirow[t]{2}{*}{$\begin{array}{l}\text { Cuervo } \\
\text { and Restrepo } \\
2007\end{array}$} & \multirow[t]{2}{*}{ Colombia } & \multirow[t]{2}{*}{$\begin{array}{l}\text { tropical } \\
\text { premontane } \\
\text { moist forest }\end{array}$} & \multirow[t]{2}{*}{$\begin{array}{l}\text { habitat } \\
\text { fragmentation }\end{array}$} & \multirow[t]{2}{*}{ population } & $\begin{array}{l}142 \text { forest } \\
\text { passerines }\end{array}$ & not specified & 1545 ind. & $\begin{array}{l}\text { outermost } \\
\text { rectrix length }\end{array}$ & & yes & yes & yes & yes $(+)^{4}$ & 0.08 \\
\hline & & & & & $\begin{array}{l}158 \text { forest } \\
\text { passerines }\end{array}$ & not specified & 1740 ind. & $\begin{array}{l}\text { tarsus } \\
\text { length }\end{array}$ & & yes & yes & yes & yes $(+)^{4}$ & 0.16 \\
\hline $\begin{array}{l}\text { Dauwe } \\
\text { et al. } 2006\end{array}$ & Belgium & $\begin{array}{l}\text { deciduous } \\
\text { parkland }\end{array}$ & $\begin{array}{l}\text { air pollution } \\
\text { (heavy metals) }\end{array}$ & population & $\begin{array}{l}\text { Parus } \\
\text { major }\end{array}$ & $\begin{array}{l}\text { fledglings/ } \\
\text { adults }\end{array}$ & 150 ind. & $\begin{array}{l}\text { outermost } \\
\text { rectrix length }\end{array}$ & & yes & yes & yes & yes $(-)$ & -0.35 \\
\hline \multirow[t]{3}{*}{$\begin{array}{l}\text { De Santo } \\
\text { et al. } 2002\end{array}$} & \multirow[t]{3}{*}{ Chile } & \multirow[t]{3}{*}{$\begin{array}{l}\text { temperate } \\
\text { rainforest }\end{array}$} & \multirow[t]{3}{*}{$\begin{array}{l}\text { habitat } \\
\text { fragmentation }\end{array}$} & \multirow[t]{3}{*}{ population } & \multirow[t]{3}{*}{$\begin{array}{l}\text { Sclerochilus } \\
\text { rubecula }\end{array}$} & \multirow[t]{3}{*}{ nestlings } & \multirow[t]{3}{*}{106 nests } & $\begin{array}{l}\text { tarsus } \\
\text { length }\end{array}$ & & no & no & no & no & $<0.10^{3}$ \\
\hline & & & & & & & & $\begin{array}{l}\text { gth } \\
\text { primary length }\end{array}$ & & no & no & no & no & $<0.10^{3}$ \\
\hline & & & & & & & & $\begin{array}{l}\text { flattened } \\
\text { wing length }\end{array}$ & & no & no & no & no & $<0.10^{3}$ \\
\hline \multirow[t]{6}{*}{$\begin{array}{l}\text { Eeva } \\
\text { et al. } 2000\end{array}$} & \multirow[t]{6}{*}{ Finland } & \multirow[t]{6}{*}{$\begin{array}{l}\text { mixed } \\
\text { forests in } \\
\text { vicinity of } \\
\text { copper smelter }\end{array}$} & \multirow[t]{6}{*}{$\begin{array}{l}\text { air pollution } \\
\text { (heavy metals) }\end{array}$} & \multirow[t]{6}{*}{ individual } & \multirow[t]{3}{*}{ Parus major } & \multirow[t]{3}{*}{ nestlings } & \multirow[t]{3}{*}{48 nests } & $\begin{array}{l}\text { 3rd } \\
\text { primary } \\
\text { length }\end{array}$ & \multirow[t]{6}{*}{$\begin{array}{l}\text { distance } \\
\text { from } \\
\text { pollution } \\
\text { source }\end{array}$} & no & yes & yes & yes $(+)$ & 0.47 \\
\hline & & & & & & & & $\begin{array}{l}\text { outermost } \\
\text { rectrix length }\end{array}$ & & no & yes & yes & no & $-{ }^{2}$ \\
\hline & & & & & & & & tarsus length & & no & yes & yes & no & $-{ }^{2}$ \\
\hline & & & & & \multirow[t]{3}{*}{$\begin{array}{l}\text { Ficedula } \\
\text { hypoleuca }\end{array}$} & \multirow[t]{3}{*}{ nestlings } & \multirow[t]{3}{*}{37 nests } & $\begin{array}{l}\text { 3rd } \\
\text { primary } \\
\text { length }\end{array}$ & & no & yes & yes & no & $-^{2}$ \\
\hline & & & & & & & & $\begin{array}{l}\text { outermost } \\
\text { rectrix length }\end{array}$ & & no & yes & yes & no & $-{ }^{2}$ \\
\hline & & & & & & & & $\begin{array}{l}\text { tarsus length } \\
\text { tarsus width }\end{array}$ & & $\begin{array}{l}\text { no } \\
\text { no }\end{array}$ & $\begin{array}{l}\text { yes } \\
\text { yes }\end{array}$ & $\begin{array}{l}\text { yes } \\
\text { yes }\end{array}$ & $\begin{array}{l}\text { yes }(+) \\
\text { no }\end{array}$ & $\begin{array}{l}0.57 \\
-2\end{array}$ \\
\hline
\end{tabular}


Table 1. Continued.

\begin{tabular}{|c|c|c|c|c|c|c|c|c|c|c|c|c|c|c|}
\hline Reference & Country & Habitat & Stress & Level & Species & Age/Sex & $\begin{array}{l}\text { \# individuals } \\
\text { or nests }\end{array}$ & Bilateral trait & $\begin{array}{l}\text { Individual } \\
\text { covariate }\end{array}$ & RM & $\overline{\mathrm{DA}}$ & AS & $\begin{array}{l}\text { Significant } \\
\text { effect }\end{array}$ & $\begin{array}{l}\text { Effect } \\
\text { size }\end{array}$ \\
\hline & & & & individual & Parus major & nestlings & 48 nests & $\begin{array}{l}\text { 3rd primary } \\
\text { length }\end{array}$ & $\begin{array}{l}\text { breeding } \\
\text { success }\end{array}$ & no & yes & yes & no & $-^{2}$ \\
\hline & & & & & & & & $\begin{array}{l}\text { outermost } \\
\text { rectrix length }\end{array}$ & & no & yes & yes & no & $--^{2}$ \\
\hline & & & & & & & & tarsus length & & no & yes & yes & yes $(-)$ & -0.46 \\
\hline & & & & & $\begin{array}{l}\text { Ficedula } \\
\text { hypoleuca }\end{array}$ & nestlings & 37 nests & & & no & yes & yes & no & \\
\hline & & & & & & & & $\begin{array}{l}\text { outermost } \\
\text { rectrix length }\end{array}$ & & no & yes & yes & no & $--^{2}$ \\
\hline & & & & & & & & tarsus length & & no & yes & yes & yes $(+)$ & 0.35 \\
\hline & & & & & & & & tarsus width & & no & yes & yes & no & \\
\hline \multirow{3}{*}{$\begin{array}{l}\text { Herrando } \\
\text { and Brotons } \\
2001\end{array}$} & Spain & $\begin{array}{l}\text { Mediterranean } \\
\text { shrubland }\end{array}$ & fire & population & $\begin{array}{l}\text { Sardinian } \\
\text { warbler }\end{array}$ & $\begin{array}{l}\text { unmoulted } \\
\text { juveniles }\end{array}$ & 24 ind. & rectrix length & & yes & yes & yes & yes $(+)$ & 0.42 \\
\hline & & & & & & $\begin{array}{l}\text { moulted } \\
\text { juveniles }\end{array}$ & 12 ind. & rectrix length & & yes & yes & yes & no & 0.23 \\
\hline & & & & & & adults & $I 1$ ind. & rectrix length & & yes & yes & yes & no & -0.30 \\
\hline $\begin{array}{l}\text { LeClerc } \\
\text { et al. } 2005\end{array}$ & USA & $\begin{array}{l}\text { golf course vs } \\
\text { grassland }\end{array}$ & $\begin{array}{l}\text { loss of native } \\
\text { vegetation } \\
\text { communities, } \\
\text { pesticides }\end{array}$ & population & Sialia sialis & nestlings & 85 nests & tarsus length & & yes & yes & yes & yes $(-)$ & -0.46 \\
\hline \multirow{10}{*}{$\begin{array}{l}\text { Lens and } \\
\text { Van Dongen } \\
1999\end{array}$} & Kenya & $\begin{array}{l}\text { tropical } \\
\text { cloud forest }\end{array}$ & $\begin{array}{l}\text { habitat } \\
\text { fragmentation }\end{array}$ & population & $\begin{array}{l}\text { Nectarinia } \\
\text { olivacea }\end{array}$ & adults & 238 ind. & tarsus length & & yes & yes & yes & yes $(+)^{5}$ & 0.38 \\
\hline & & & & & & & 193 ind. & pectoral tuft & & yes & yes & yes & & 0.46 \\
\hline & & & & & $\begin{array}{l}\text { Andropadus } \\
\text { milanjensis }\end{array}$ & adults & 98 ind. & tarsus length & & yes & yes & yes & & 0.53 \\
\hline & & & & & & & 89 ind. & $\begin{array}{l}\text { cheek patch } \\
\text { width }\end{array}$ & & yes & yes & yes & & 0.41 \\
\hline & & & & & $\begin{array}{l}\text { Pogonocichla } \\
\text { stellata }\end{array}$ & adults & 269 ind. & tarsus length & & yes & yes & yes & & 0.40 \\
\hline & & & & & & & 141 ind. & $\begin{array}{l}\text { pre-ocular } \\
\text { spot width }\end{array}$ & & yes & yes & yes & & 0.39 \\
\hline & & & & & & & 84 ind. & $\begin{array}{l}\text { pre-ocular } \\
\text { spot height }\end{array}$ & & yes & yes & yes & & 0.61 \\
\hline & & & & & $\begin{array}{l}\text { Zoserops } \\
\text { silvanus }\end{array}$ & adults & 122 ind. & tarsus length & & yes & yes & yes & & 0.50 \\
\hline & & & & & & & 104 ind. & eye ring width & & yes & yes & yes & & 0.32 \\
\hline & & & & & & & 104 ind. & eye ring height & & yes & yes & yes & & 0.32 \\
\hline
\end{tabular}


Table 1. Continued.

\begin{tabular}{|c|c|c|c|c|c|c|c|c|c|c|c|c|c|c|}
\hline Reference & Country & Habitat & Stress & Level & Species & Age/Sex & $\begin{array}{l}\text { \# individuals } \\
\text { or nests }\end{array}$ & Bilateral trait & $\begin{array}{l}\text { Individual } \\
\text { covariate }\end{array}$ & RM & $\overline{\mathrm{DA}}$ & AS & $\begin{array}{l}\text { Significant } \\
\text { effect }\end{array}$ & $\begin{array}{l}\text { Effect } \\
\text { size }\end{array}$ \\
\hline & & & & & $\begin{array}{l}\text { Turdus } \\
\text { helleri }\end{array}$ & adults & 91 ind. & tarsus length & & yes & yes & yes & & 0.67 \\
\hline & & & & & & & 96 ind. & $\begin{array}{l}\text { post-ocular } \\
\text { patch width }\end{array}$ & & yes & yes & yes & & 0.37 \\
\hline & & & & & & & 69 ind. & rextric length & & yes & yes & yes & & 0.63 \\
\hline \multirow[t]{9}{*}{$\begin{array}{l}\text { Lens et al. } \\
2000\end{array}$} & \multirow[t]{9}{*}{ Kenya } & \multirow[t]{9}{*}{$\begin{array}{l}\text { tropical } \\
\text { cloud forest }\end{array}$} & \multirow[t]{9}{*}{$\begin{array}{l}\text { habitat } \\
\text { fragmentation }\end{array}$} & \multirow[t]{9}{*}{ individual } & \multirow[t]{9}{*}{$\begin{array}{l}\text { Turdus } \\
\text { helleri }\end{array}$} & \multirow[t]{9}{*}{ adults } & 18 ind. & $\begin{array}{l}\text { tarsus length } \\
\text { (pop. I) }\end{array}$ & \multirow[t]{9}{*}{ inbreeding } & yes & yes & yes & yes $(+)^{6}$ & 0.60 \\
\hline & & & & & & & 67 ind. & $\begin{array}{l}\text { rextrix length } \\
\text { (pop. 1) }\end{array}$ & & yes & yes & yes & & 0.54 \\
\hline & & & & & & & 152 ind. & $\begin{array}{l}\text { postocular } \\
\text { patch width } \\
\text { (pop. I) }\end{array}$ & & yes & yes & yes & & 0.26 \\
\hline & & & & & & & 14 ind. & $\begin{array}{l}\text { tarsus length } \\
\text { (pop. 2) }\end{array}$ & & yes & yes & yes & yes $(+)^{6}$ & 0.22 \\
\hline & & & & & & & 53 ind. & $\begin{array}{l}\text { rextrix length } \\
\text { (pop. 2) }\end{array}$ & & yes & yes & yes & & 0.20 \\
\hline & & & & & & & 102 ind. & $\begin{array}{l}\text { postocular } \\
\text { patch width } \\
\text { (pop. 2) }\end{array}$ & & yes & yes & yes & & 0.17 \\
\hline & & & & & & & 15 ind. & $\begin{array}{l}\text { tarsus length } \\
\text { (pop. 3) }\end{array}$ & & yes & yes & yes & $\mathrm{no}^{6}$ & 0.00 \\
\hline & & & & & & & 58 ind. & $\begin{array}{l}\text { rextrix length } \\
\text { (pop. 3) }\end{array}$ & & yes & yes & yes & & 0.00 \\
\hline & & & & & & & 122 ind. & $\begin{array}{l}\text { postocular } \\
\text { patch width } \\
\text { (pop. 3) }\end{array}$ & & yes & yes & yes & & 0.00 \\
\hline $\begin{array}{l}\text { Lens et al. } \\
2002\end{array}$ & Kenya & $\begin{array}{l}\text { tropical } \\
\text { cloud forest }\end{array}$ & $\begin{array}{l}\text { habitat } \\
\text { fragmentation }\end{array}$ & individual & $\begin{array}{l}\text { Turdus } \\
\text { helleri }\end{array}$ & adults & 18 ind. & tarsus length & survival & yes & yes & yes & yes (+) & 0.49 \\
\hline \multirow[t]{2}{*}{$\begin{array}{l}\text { Maul and } \\
\text { Farris 2005 }\end{array}$} & \multirow[t]{2}{*}{ USA } & $\begin{array}{l}\text { agricultural } \\
\text { fields } v s\end{array}$ & \multirow[t]{2}{*}{$\begin{array}{l}\text { cholinesterase- } \\
\text { inhibiting } \\
\text { pesticides }\end{array}$} & \multirow[t]{2}{*}{ population } & \multirow[t]{2}{*}{$\begin{array}{l}\text { Cardinalis } \\
\text { cardinalis }\end{array}$} & \multirow[t]{2}{*}{ not specified } & \multirow[t]{2}{*}{82 ind. } & tarsus width & & yes & yes & yes & yes $(+)$ & 0.16 \\
\hline & & forest/wetlands & & & & & & $\begin{array}{l}\text { unflatted } \\
\text { wing length }\end{array}$ & & & & & & \\
\hline \multirow[t]{3}{*}{ Møller 1995} & \multirow[t]{3}{*}{ Denmark } & \multirow[t]{3}{*}{ Farmland } & \multirow[t]{3}{*}{$\begin{array}{l}\text { habitat } \\
\text { fragmentation }\end{array}$} & individual & $\begin{array}{l}\text { Turdus } \\
\text { merula }\end{array}$ & nestlings & 51 ind. & \multirow{3}{*}{$\begin{array}{l}\text { flattened wing } \\
\text { length } \\
\text { outermost } \\
\text { rectrix length } \\
\text { flattened wing } \\
\text { length }\end{array}$} & \multirow[t]{3}{*}{$\begin{array}{l}\text { post-fledgling } \\
\text { survival }\end{array}$} & no & yes & yes & yes $(+)$ & 0.75 \\
\hline & & & & & & & & & & no & yes & yes & yes $(+)$ & 0.76 \\
\hline & & & & population & $\begin{array}{l}\text { Turdus } \\
\text { merula }\end{array}$ & nestlings & 61 ind. & & & no & yes & yes & yes $(+)$ & 0.35 \\
\hline
\end{tabular}


Table 1. Continued.

\begin{tabular}{|c|c|c|c|c|c|c|c|c|c|c|c|c|c|c|}
\hline Reference & Country & Habitat & Stress & Level & Species & Age/Sex & $\begin{array}{l}\text { \# individuals } \\
\text { or nests }\end{array}$ & Bilateral trait & $\begin{array}{l}\text { Individual } \\
\text { covariate }\end{array}$ & RM & $\overline{D A}$ & $\overline{A S}$ & $\begin{array}{l}\text { Significant } \\
\text { effect }\end{array}$ & $\begin{array}{l}\text { Effect } \\
\text { size }\end{array}$ \\
\hline & & & & & & & & $\begin{array}{l}\text { outermost } \\
\text { rectrix length }\end{array}$ & & no & yes & yes & yes $(+)$ & 0.30 \\
\hline & & & & & & fledglings & 20 ind. & & & no & yes & yes & no & 0.41 \\
\hline & & & & & & & & & & no & yes & yes & no & 0.26 \\
\hline & & & & & & adults & 41 ind. & $\begin{array}{l}\text { outermost } \\
\text { rectrix length }\end{array}$ & & no & yes & yes & no & 0.18 \\
\hline & & & & & & & & $\begin{array}{l}\text { outermost } \\
\text { rectrix length }\end{array}$ & & no & yes & yes & no & 0.12 \\
\hline $\begin{array}{l}\text { Pérez-Tris } \\
\text { et al. } 2000\end{array}$ & Spain & $\begin{array}{l}\text { mixed forest } \\
\text { along drought } \\
\text { gradient }\end{array}$ & $\begin{array}{l}\text { distributional } \\
\text { edge effects }\end{array}$ & population & $\begin{array}{l}\text { Erithacus } \\
\text { rubecula }\end{array}$ & $\begin{array}{l}\text { fledglings/ } \\
\text { adults }\end{array}$ & 112 ind. & tarsus length & & yes & yes & yes & no & 0.09 \\
\hline $\begin{array}{l}\text { Pérez-Tris } \\
\text { et al. } 2002\end{array}$ & Spain & oak forest & parasites & individual & $\begin{array}{l}\text { Sylvia } \\
\text { atricapilla }\end{array}$ & $\begin{array}{l}\text { fledglings/ } \\
\text { adults }\end{array}$ & 76 ind. & rectrix length & mite load & yes & no & no & yes $(+)$ & 0.30 \\
\hline
\end{tabular}

${ }^{1}$ No significant relationships in two other populations and in females

${ }^{2}$ Statistics did not allow (more accurate) estimation of effect sizes

${ }^{3}$ Not significant after Bonferroni correction

${ }^{4}$ Heterogenous among species

${ }^{5}$ Species and traits were treated as random effects in a single statistical model

${ }^{6}$ Different traits were treated as repeated measurements 
parasites or severe weather conditions. Traits were measured on both juveniles (nestling and fledgling stages) and adults, and comprised various measures of wing, tail and tarsus length or specific feather measurements (e.g. weight and density). Only two studies (Lens and Van Dongen 1999, Lens et al. 2000) included secondary sexual traits (eye-rings, supercilia, length of tail streamers). While most studies met the basic statistical requirements of repeated measurements and correction for DA and AS, only three studies (Lens and Van Dongen 1999, Lens et al. 2000, Maul and Farris 2005) combined information across different traits. The majority of studies compared FA estimates at population level, while two studies (Anciães and Marini 2000, Cuervo and Restrepo 2007) additionally studied assemblage-level patterns. Nine studies also examined relationships at the level of individuals, thereby relating FA estimates with measures of body condition, survival and genetic inbreeding.

In 13 studies, relationships between individual or population FA and the variable(s) of interest were statistically significant and in the expected direction. In seven other studies, relationships were not statistically significant or heterogeneous among traits, ages or sexes, while in one study relationships were statistically significant in the opposite direction. Positive effect sizes ranged between 0.02 and 0.76 (weighted average \pm SD: $0.30 \pm 0.17$ ).

\section{Discussion and conclusion}

Since the publication of the milestone papers by Leary and Allendorf (1989) and Clarke (1995), it has become clear that the use of FA as a universal biomarker in conservation biology is hampered by various conceptual and methodological problems. Most importantly, the underlying mechanisms of developmental homeostasis remain poorly known, despite substantial improvement in the statistical treatment of asymmetry data. It is therefore impossible to predict when, and to what extent, FA may be a reliable estimator of the underlying developmental instability, which is key to detecting relationships with other variables of interest. In most case studies listed in Table 1 , observed heterogeneity in relationships with FA (between ages, traits or stressors) was explained by the authors, thereby addressing various methodological and conceptual issues such as unequal statistical power in testing relationships among age groups (Herrando and Brotons 2001), presumed differences in level of directional selection between sexes (Møller 1993) and presumed differences in pathways through which stress affected trait development (e.g. sulphuric oxides and heavy metals directly affecting calcium metabolism of tarsi: Eeva et al. 2000). Studies that failed to detect predicted relationships with FA either reported significant (yet variable) effects on other phenotypic or life-history measures (e.g. body mass, ptilochronology, reproductive success, survival: Carbonell and Tellería 1999, Pérez-Tris et al. 2000, De Santo et al. 2002, Carbonell et al. 2003) or did not quantify stress effects at all (e.g. Dauwe et al. 2006). As may be expected from studies on natural, free-ranging populations, presumed stress effects were not a priori validated by independent measures.

The majority of studies examined met the basic statistical requirements of FA analysis (appropriate sample sizes, repeated measurements, tests for DA and AS), although one study (Herrando and Brotons 2001) ascribed between-group heterogeneity in relationships between FA and stress to unequal sample sizes. Roughly half of the studies measured more than one trait per individual, but only three studies combined information across traits, either by calculating a composite FA index (Maul and Farris 2005) or by treating multiple traits as repeated measurements at the level of the individual (Lens and Van Dongen, 1999, Lens et al. 2000). When organism-wide asymmetry (Individual Asymmetry Parameter, IAP; Clarke 1998) is expected, analyses that combine information across traits are believed to be more powerful in detecting relationships with stress and fitness than single-trait analyses (Leary and Allendorf 1989, Watson and Thornhill 1994, Leung et al. 2000). Individual-level concordance in FA is, however, rarely observed in field studies (Dufour and Weatherhead 1996, Sarre 1996, Lens and Van Dongen 1999, Anciães and Marini 2000), although it is often implicitly assumed in evolutionary models of FA. This seems a general trend, even for traits that are correlated 
developmentally (but see Atchley et al. 1984, Leamy and Atchley 1984, Leamy 1993 for supportive cases). Absence of IAPs may be due to trait-specific timing during ontogeny when environmental perturbations can cause aberrant phenotypes (Clarke 1995), or to low repeatability of FA at the individual (as opposed to population) level resulting from high sampling variability or proportionally large measurement error (Whitlock 1996, Van Dongen 1998). In contrast, populations with high levels of FA in one trait were often strongly asymmetric in other traits too (Population Asymmetry Parameter; Clarke 1998). Given that prior knowledge on the level of stress sensitivity of a particular trait is nearly always lacking in applied conservation studies, it seems prudent to measure as many bilateral traits as possible when handling individuals, and apply rigorous statistical techniques to assess the level of between-trait correlation in FA, afterwards. Based on the outcome of these explorative tests, researchers can then decide whether or not to combine information across traits when testing relationships with the variable(s) of interest.

Several studies (e.g. Whitlock 1996, Houle 1997) showed that developmental stability estimated by FA has a very low repeatability (i.e. single-trait FA typically owes only $7 \%$ of its variance to underlying individual differences in developmental stability; Gangestad and Thornhill 1999). Owing to sampling variability and the random nature of the developmental processes that generate FA, individuals with identical underlying levels of stability can therefore be expected to express different levels of FA (Whitlock 1998) and developmentally "unstable" individuals may even display lower FA levels than "stable" ones, despite their poorer intrinsic quality (Lens et al. 2002a; see also Palmer 1994, Leung and Forbes 1997). Nevertheless, twothirds of the case studies examined in this paper reported significant, positive relationships between anthropogenic stress effects and levels of FA. Effect sizes ranged from very small (0.02) to very large $(0.76)$, with a weighted average of 0.30 across all reported (positive) relationships (indicating that on average $9 \%$ of the variance in the variable of interest was explained by variation in FA). Following Cohen (1988), such value corresponds to a "medium effect". Møller and Jennions (2002) reported that more than $80 \%$ of $r^{2}$-values derived from eight meta-analyses of ecological and evolutionary studies were also smaller than 10\%, and subsequent analysis showed that average effect sizes in studies of FA were not significantly smaller than in other ecological or evolutionary studies. As relationships between FA and developmental stability are generally believed to be more reliable under high than under low stress (Lens et al. 2002a), elevated levels of environmental stress inherent to most conservation studies may (partly) explain the higher incidence of significant FA-stress and FA-fitness relationships in this review, compared to others (e.g. Bjorksten et al. 2000).

\section{Towards an integrated study of biomarkers}

Based on the selection of studies examined in this paper, avian ecologists seem to apply FA more often at the population than at the individual level. In addition, threatened populations can be expected to be exposed to substantial levels of exogenous (or endogenous) stress. Under such conditions, both sampling error and statistical noise, owing to the random nature of developmental processes, can be expected to be rather low, and chances of detecting biologically significant relationships with FA may hence be reasonable. Nevertheless, whenever possible within the tight logistic framework of an applied conservation study, it remains highly recommended to integrate different types of individual-based biomarkers. One promising candidate biomarker to combine with FA is ptilochronology (Grubb 1989, 2006), i.e. the study of alternating dark and light growth-bars on bird feathers. Each pair of growth-bars on a bird's feather constitutes a 24-hour period of growth, and growth-bar patterns are believed to reflect the past nutritional condition of an individual. Since the introduction of the concept of ptilochronology, a growing number of correlative and experimental studies have investigated relationships between growth-bar dimensions and stress, while others explored ways in which nutritional condition, as indexed by the rate of feather growth, can be used as a measure of 
fitness. The fact that feathers grow multiple times from the same follicle (i.e. after each natural or induced moult) suggests that their growth parameters (rate or precision) may allow the tracking of environmental (nutritional) stress conditions throughout an individual's life span (i.e. as opposed to tarsus measures) (Swaddle 1997, Aparicio 2001, Stige et al. 2005). As 16 studies reviewed in this paper effectively measured one or more properties of wing or tail feathers, a complementary study of growth-bars could have been integrated without much extra effort. Three papers that did so (Carbonell and Tellería 1999, Pérez-Tris et al. 2000, Carbonell et al. 2003) revealed stronger or more consistent relationships with ptilochronology than with FA (see Møller 1996, Talloen et al. in press for other examples).

In conclusion, during the last decade ecologists have continued to study FA as a biomarker of anthropogenic stress across a wide diversity of habitats and biogeographical regions, despite the lack of a theoretical framework that predicts under which environmental conditions biologically significant relationships between FA, stress and fitness can be expected. At the same time, ptilochronology has been increasingly, and successfully, applied in different conservation hotspots, i.e. Jamaica (Strong and Sherry 2000), Brazil (Stratford and Stouffer 2001), Peru (Velando 2002), Australia (Doucet and Montgomerie 2003) and Kenya (Spanhove and Lens unpubl. data). Owing to their repeated and tractable growth history, bird feathers hence seem to comprise a highly appropriate trait for integrated studies that measure both their rate of growth, through ptilochronology, and precision of growth, through FA, when aiming to predict how anthropogenic factors may affect the future viability of birds of conservation concern.

\section{Acknowledgements}

We are grateful to M. Anciães, J. Bustnes, C. R. Brown, T. Dauwe, T. Eeva, A. P. Møller, J. Pérez-Tris and J. Swaddle for additional comments on their studies. Two anonymous referees provided helpful comments on an earlier draft. HE is a postdoctoral fellow with the Fund for Scientific Research, Flanders (FWO-Vlaanderen). This study was funded by FWO research project G.0210.04.

\section{References}

Alibert, P. and Auffray, J. C. (2003) Genomic coadaptation, outbreeding depression and developmental instability. Pp. 116-134 in M. Polak, ed. Developmental instability: causes and consequences. New York: Oxford University Press.

Anciães, M. and Marini, M. A. (2000) The effects of fragmentation on fluctuating asymmetry in passerine birds of Brazilian tropical forests. J. Appl. Ecol. 37: 1013-1028.

Aparicio, J. M. (2001) Patterns of growth and fluctuating asymmetry: the effects of asymmetrical investment in traits with determinate growth. Behav. Ecol. Sociobiol. 49: 273-282.

Atchley, W. R., Herring, S. W., Riska, B. and Plummer, A. A. (1984) Effects of the muscular dysgenesis gene on developmental stability in the mouse mandible. J. Cran. Gen. Devel. Biol. 4: 179-189.

Auffray, J. C., Debat, V. and Alibert, P. (1999) Shape asymmetry and developmental stability. Pp. 309-324 in M. A. J. Chaplain, G. D. Singh and J. C. McLachlan, eds. On growth and form: spatio-temporal pattern formation in biology. Chichester: John Wiley and Sons.

Begg, C. B. (1994) Publication bias. Pp. 399-409 in H. Cooper and L. V. Hedges, eds. The handbook of research synthesis. New York: Russel Sage Foundation.

Bergman, K.-O., Askling, J., Ekberg, O., Ignell, H., Wahlman, H. and Milberg, P. (2004) Landscape effects on butterfly assemblages in an agricultural region. Ecography 27: 619-628. 
Bjordal, H. (1983) Effects of deep freezing, freeze-drying and skinning on body dimensions of house sparrows Passer domesticus. Fauna Norv. Ser. C 6: 105-108.

Bjorksten, T. A., Fowler, K. and Pomiankowski, A. (2000) What does sexual trait FA tell us about stress? Trends Ecol. Evol. 15: 163-166.

Brown, C. R. and Brown, M. B. (1998) Intense natural selection on body size and wing and tail asymmetry in cliff swallows during severe weather. Evolution 52: 1461-1475.

Brown, C. R. and Brown, M. B. (1999) Natural selection on tail and bill morphology in Barn Swallows Hirundo rustica during severe weather. Ibis 141: 652-659.

Burke, D. and Nol, E. (2001) Age ratios and return rates of adult male Ovenbirds in contiguous and fragmented forests. J. Field Orn. 72: 433-438.

Bustnes, J. O., Folstad, I., Erikstad, K. E., Fjeld, M., Miland, O. O. and Skaare, J. U. (2002) Blood concentration of organochlorine pollutants and wing feather asymmetry in Glaucous Gulls. Funct. Ecol. 16: 617-622.

Bustnes, J. O., Kristiansen, K. O. and Helberg, M. (2007) Immune status, carotenoid coloration, and wing feather growth in relation to organochlorine pollutants in Great Black-backed Gulls. Arch. Environ. Contam. Toxicol. 53: 96-102.

Carbonell, R., Pérez-Tris, J. and Tellería, J. L. (2003) Effects of habitat heterogeneity and local adaptation on the body condition of a forest passerine at the edge of its distributional range. Biol. J. Linn. Soc. 78: 479-488.

Carbonell, R. and Tellería, J. L. (1998) Increased asymmetry of tarsus-length in three populations of Blackcaps Sylvia atricapilla as related to proximity to range boundary. Ibis 140: 331-333.

Carbonell, R. and Tellería, J. L. (1999) Feather traits and ptilochronology as indicators of stress in Iberian Blackcaps Sylvia atricapilla. Bird Study 46: 243-248.

Chave, J. and Norden, N. (2007) Changes of species diversity in a simulated fragmented neutral landscape. Ecol. Model. 207: 3-10.

Clarke, G. M. (1993) Fluctuating asymmetry of invertebrate populations as a biological indicator of environmental quality. Environ. Pollut. 82: 207-211.

Clarke, G. M. (1995) Relationships between developmental stability and fitness: application for conservation biology. Conserv. Biol. 9: 18-24.

Clarke, G. M. (1998) The genetic basis of developmental stability. IV. Individual and population asymmetry parameters. Heredity 80: 553-561.

Clarke, G. M. and McKenzie, L. J. (1992) Fluctuating asymmetry as a quality control indicator for insect mass rearing processes. J. Econom. Entomol. 85: 2045-2050.

Clarke, G. M., Oldroyd, B. P. and Hunt, P. (1992) The genetic basis of developmental stability in Apis mellifera: heterozygosity vs. genic balance. Evolution 46: 753-762.

Cohen, J. (1988) Statistical power analysis for the behavioral sciences. Second edition. Hillsdale, N.J: Erlbaum.

Cuervo, A. M. and Restrepo, C. (2007) Assemblage and population-level consequences of forest fragmentation on bilateral asymmetry in tropical montane birds. Biol. J. Linn. Soc. 92: 119-133.

Dauwe, T., Janssens, E. and Eens, M. (2006) Effects of heavy metal exposure on the condition and health of adult Great Tits (Parus major). Environ. Pollut. 140: 71-78.

De Santo, T. L., Willson, M. F., Sieving, K. E. and Armesto, J. J. (2002) Nesting biology of tapaculos (Rhinocryptidae) in fragmented south-temperate rainforests of Chile. Condor 104: 482-495.

Diekotter, T., Haynes, K. J., Mazeffa, D. and Crist, T. O. (2007) Direct and indirect effects of habitat area and matrix composition on species interactions among flowervisiting insects. Oikos 116: 1588-1598.

Doucet, S. M. and Montgomerie, R. (2003) Multiple sexual ornaments in satin bowerbirds: ultraviolet plumage and bowers signal different aspects of male quality. Behav. Ecol. 14: 503-509.

Dufour, K. W. and Weatherhead, P. J. (1996) Estimation of organism-wide asymmetry in red-winged blackbirds and its relation to studies of mate selection. Proc. R. Soc. Lond. B. 263: 769-775.

Eeva, T., Tanhuanpää, S., Råbergh, C., Airaksinen, S., Nikinmaa, M. and 
Lehikoinen, E. (2000) Biomarkers and fluctuating asymmetry as indicators of pollution-induced stress in two hole-nesting passerines. Funct. Ecol. 14: 235-243.

Erbout, N., De Meyer, M. and Lens, L. (in press) Hybridisation between two polyphagous fruit fly species (Diptera: Tephritidae) causes sex-biased reduction in developmental stability. Biol. J. Linn. Soc.

Fiering, S., Whitelaw, E. and Martin, D. I. K. (2000) To be or not to be active: the stochastic nature of enhancer action. BioEssays 22: 381-387.

Fischer, J. and Lindenmayer, D. B. (2007) Landscape modification and habitat fragmentation: a synthesis. Global Ecol. Biogeogr. 16: 265-280.

Gangestad, S. W. and Thornhill, R. (1999) Individual differences in developmental precision and fluctuating asymmetry: a model and its implications. J. Evol. Biol. 12: 402-416.

Graham, J. H. (1992) Genomic coadaptation and developmental stability in hybrid zones. Acta Zool. Fenn. 191: 121-131.

Graham, J. H., Freeman, D. C. and Emlen, J. M. (1993) Antisymmetry, directional asymmetry, and dynamic morphogenesis. Genetica 89: 121-137.

Grubb, T. C. (1989) Ptilochronology: feather growth bars as indicators of nutritional status. Auk 106: 314-320.

Grubb, T. C. (2006) Ptilochronology: feather time and the biology of birds. New York: Oxford University Press (Oxford Ornithological Series).

Habib, L., Bayne, E. M. and Boutin, S. (2007) Chronic industrial noise affects pairing success and age structure of ovenbirds Seiurus aurocapilla. J. Appl. Ecol. 44: $176-184$.

Hendrickx, F., Maelfait, J.-P., van Wingerden, W., Schweiger, O., Speelmans, M., Aviron, S., Augenstein, I., Billeter, R., Bailey, D., Bukacek, R., Burel, F., Diekotter, T., Dirksen, J., Herzog, F., Liira, J., Roubalova, M., Vandomme, V. and Bugter, R. (2007) How landscape structure, land-use intensity and habitat diversity affect components of total arthropod diversity in agricultural landscapes. J. Appl. Ecol. 44: 340-351.
Herrando, S. and Brotons, L. (2001) Fluctuating asymmetry in Sardinian Warblers Sylvia melanocephala inhabiting two shrublands affected by fire. Bird Study 48: $180-187$.

Holmes, R. T., Marra, P. P. and Sherry, T. W. (1996) Habitat-specific demography of breeding black-throated blue warblers (Dendroica caerulescens): implications for population dynamics. J. Anim. Ecol. 65: 183-195.

Houle, D. (1997) Comment on "A metaanalysis of the heritability of developmental stability" by Møller and Thornhill. J. Evol. Biol. 10: 17-20.

Huggett, R. J., Kimerle, R. A., Mehrle, P. M. J. and Bergman, H. L. (1992) Biomarkers: biochemical, physiological, and histological markers of anthropogenic stress. Boca Raton, Florida: Lewis Publishers.

Kark, S., Safriel, U. N., Tabarroni, C. and Randi, E. (2001) Relationship between heterozygosity and asymmetry: a test across the distribution range. Heredity 86: 119-127.

Karvonen, E., Merilä, J., Rintamäki, P. T. and Van Dongen, S. (2003) Geography of fluctuating asymmetry in the greenfinch, Carduelis chloris. Oikos 100: 507-516.

Kieser, J. A. and Groeneveld, H. T. (1991) Fluctuating odontometric asymmetry, morphological variability, and genetic monomorphism in the cheetah Acinonyx jubatus. Evolution 45: 1175-1183.

Klingenberg, C. P. (2001) A developmental perspective on developmental instability: theory, models and mechanisms. Pp. 14-34 in M. Polak, ed. Developmental instability: causes and consequences. Oxford: Oxford University Press.

Klingenberg, C. P. and Nijhout, H. F. (1999) Genetics of fluctuating asymmetry: a developmental model of developmental instability. Evolution 53: 358-375.

Kuhn, T. S. (1970) The structure of scientific revolutions. Chicago: University of Chicago Press.

Leamy, L. (1993) Morphological integration of fluctuating asymmetry in the mouse mandible. Genetica 89: 139-153.

Leamy, L. (1999) Heritability of directional and fluctuating asymmetry for mandibular 
characters in random-bred mice. J. Evol. Biol. 12: 146-155.

Leamy, L. and Atchley, W. R. (1984) Morphometric integration in the rat (Rattus sp.) scapula. J. Zool. 202: 43-56.

Leary, R. F. and Allendorf, F. W. (1989) Fluctuating asymmetry as an indicator of stress: implications for conservation biology. Trends Ecol. Evol. 4: 214-217.

Leary, R. F., Allendorf, F. W. and Knudsen, K. L. (1983) Developmental stability and enzyme heterozygosity in rainbow trout. Nature 301: 71-72.

Leary, R. F., Allendorf, F. W. and Knudsen, K. L. (1984) Superior developmental stability of heterozygosity in enzyme loci in salmonid fishes. Amer. Nat. 124: 540-551.

Leary, R. F., Allendorf, F. W. and Knudsen, K. L. (1985) Inheritance of meristic variation and the evolution of developmental stability in rainbow trout. Evolution 39: 1318-1326.

LeClerc, J. E., Che, J. P. K., Swaddle, J. P. and Cristol, D. A. (2005) Reproductive success and developmental stability of Eastern Bluebirds on golf courses: evidence that golf courses can be productive. Wildl. Soc. Bull. 33: 483-493.

Lens, L. and Van Dongen, S. (1999) Evidence for organism-wide asymmetry in five bird species of a fragmented afrotropical forest. Proc. R. Soc. Lond. $B$ 266: 1055-1060.

Lens, L. and Van Dongen, S. (2000) Fluctuating and directional asymmetry in natural bird populations exposed to different levels of habitat disturbance, as revealed by mixture analysis. Ecol. Lett. 3: 516-522.

Lens, L., Van Dongen, S., Galbusera, P., Schenck, T., Matthysen, E. and Van de Casteele, T. (2000) Developmental instability and inbreeding in natural bird populations exposed to different levels of habitat disturbance. J. Evol. Biol 13: 889-896.

Lens, L., Van Dongen, S., Kark, S. and Matthysen, E. (2002a) Fluctuating asymmetry as an indicator of fitness: can we bridge the gap between studies? Biol. Review 77: 27-38.

Lens, L., Van Dongen, S., Kark, S., Talloen, W., Hens, L. and Matthysen, E. (2001) The use of bilateral asymmetry in ecology and conservation: Concept, developments and prospects. Pp. $21-43$ in S. G. Pandalai, ed. Recent research developments in ecology. Trivandrum: Transworld Research Network.

Lens, L., Van Dongen, S. and Matthysen, E. (2002b) Fluctuating asymmetry as an early warning system in the critically endangered Taita Thrush. Conserv. Biol. 13: 479-487.

Lens, L., Van Dongen, S., Norris, K., Githiru, M. and Matthysen, E. (2002c) Avian persistence in fragmented rainforest. Science 298: 1236-1238.

Lens, L., Van Dongen, S., Wilder, C. M., Brooks, T. M. and Matthysen, E. (1999) Fluctuating asymmetry increases with habitat disturbance in seven bird species of a fragmented afrotropical forest. Proc. $R$. Soc. Lond. B 266: 1241-1246.

Leung, B. and Forbes, M. R. (1996) Fluctuating asymmetry in relation to stress and fitness: effects of trait type as revealed by meta-analysis. Ecoscience 3: 400-413.

Leung, B. and Forbes, M. R. (1997) Modelling fluctuating asymmetry in relation to stress and fitness. Oikos 78: 397-405.

Leung, B., Forbes, M. R. and Houle, D. (2000) Fluctuating asymmetry as a bioindicator of stress: comparing efficacy of analyses involving multiple traits. Am. Nat. 155: 101-115.

Leung, B., Knopper, L. and Mineau, P. (2001) A critical assessment of the utility of fluctuating asymmetry as a biomarker of anthropogenic stress. Pp. 415-426 in M. Polak, ed. Developmental instability: causes and consequences. Oxford: Oxford University Press.

Light, R. J. and Pillemer, D. B. (1984) Summing up: the science of reviewing research. Cambridge, Mass: Harvard University Press.

Ludwig, W. (1932) Das Rechts-Links Problem im Tierreich und beim Menschen. Berlin: Springer.

Maul, J. D. and Farris, J. L. (2005) Monitoring exposure of northern cardinals, Cardinalis cardinalis, to cholinesterase-inhibiting pesticides: enzyme activity, reactivations, and indicators of environmental stress. Environ. Toxicol. Chem. 24: 1721-1730. 
Mayer, F. L., Versteeg, D. J., Mckee, M. J., Folmar, L. C., Graney, R. L., McCume, D. C. and Rattner, B. A. (1992) Physiological and nonspecific biomarkers. Pp. 5-86 in Huggett, R. J., Kimerle, R. A., Mehrle, P. M. Jr and Bergman, H. L., eds. Biomarkers: Biochemical, physiological and histological markers of anthropogenic stress. Boca Raton: Lewis Publishers.

McAdams, H. H. and Arkin, A. (1999) It's a noisy business! Genetic regulation at the nanomolecular scale. Trends Genet. 15: 65-69.

Møller, A. P. (1993) Morphology and sexual selection in the barn swallow Hirundo rustica in Chernobyl, Ukraine. Proc. $R$. Soc. Lond. B 252: 51-57.

Møller, A. P. (1995) Developmental stability and ideal despotic distribution of Blackbirds in a patchy environment. Oikos 72: 228-234.

Møller, A. P. (1996) Development of fluctuating asymmetry in tail feathers of the barn swallow Hirundo rustica. J. Evol. Biol. 9: 677-694.

Møller, A. P. (1997) Developmental stability and fitness: A review. Am. Nat. 149: 916-932.

Møller, A. P. and Jennions, M. D. (2002) How much variance can be explained by ecologists and evolutionary biologists? Oecologia 132: 492-500.

Møller, A. P. and Swaddle, J. P. (1997) Asymmetry, developmental stability, and evolution. Oxford: Oxford University Press.

Palmer A. R (1994) Fluctuating asymmetry: a primer. Pp. 335-364 in and T. A. Markow, ed. Developmental instability: its origins and evolutionary implications. Dordrecht: Kluwer Academic Publishers.

Palmer, A. R. (1996) Walzing with asymmetry: is fluctuating asymmetry a powerful new tool for biologists or just an alluring new dance step? BioScience 46: 518-532.

Palmer, A. R. and Strobeck, C. (1992) Fluctuating asymmetry as a measure of developmental stability: implications of non-normal distributions and power of statistical tests. Acta Zool. Fenn. 191: 57-72.

Palmer, A. R., Strobeck, C. and Chippindale, A. K. (1994) Bilateral variation and the evolutionary origin of macroscopic asymmetries. Pp. 203-220 in T. A. Markow, ed. Developmental instability: its origins and evolutionary implications. Dordrecht: Kluwer Academic Publishers.

Parsons, P. A. (1990) Fluctuating asymmetry: an epigenic measure of stress. Biol. Review 65: 131-154.

Parsons, P. A. (1992) Fluctuating asymmetry: a biological monitor of environmental and genomic stress. Heredity 68: 361-364.

Pérez-Tris, J., Carbonell, R. and Telleria, J. L. (2000) Abundance distribution, morphological variation and juvenile condition of Robins, Erithacus rubecula (L.), in their Mediterranean range boundary. J. Biogeogr. 27: 879-888.

Pérez-Tris, J., Carbonell, R. and Telleria, J. L. (2002) Parasites and the blackcap's tail: implications for the evolution of feather ornaments. Biol. J. Linn. Soc. 76: 481-492.

Polak, M. and Trivers, R. (1994) The science of symmetry in biology. Trends Ecol. Evol. 9: 122-124.

Reeve, E. C. R. (1960) Some genetic tests on asymmetry of sternopleural chaetae number in Drosophila. Genet. Res. 1: 151-172.

Rosenthal, R. (1991) Meta-analytic procedures for social research. Newbury Park, CA: Sage.

Sarre, S. (1996) Habitat fragmentation promotes fluctuating asymmetry but not morphological divergence in two geckos. Res. Pop. Ecol. 38: 57-64.

Schwarzer, R. (1989) META-ANALYSIS 5.3, manual (http://www.fu-berlin.de/gesund/ gesu_engl/meta_e.htm).

Simmons, L. W., Tomkins, J. L., Kotiaho, J. S. and Hunt, J. (1999) Fluctuating paradigm. Proc. R. Soc. Lond. B 266: 593-595.

Soulé, M. E. (1979) Heterozygosity and developmental stability: another look. Evolution 33: 396-401.

Stige, L. C., Slagsvold, T. and Vollestad, L. A. (2005) Individual fluctuating asymmetry in pied flycatchers (Ficedula hypoleuca) persists across moults, but is not heritable and not related to fitness. Evol. Ecol. Res. 7: $381-406$.

Stratford, J. A. and Stouffer, P. C. (2001) Reduced feather growth rates of two common birds inhabiting Central 
Amazonian forest fragments. Conserv. Biol. 15: 721-728.

Strong, A. M. and Sherry, T. W. (2000) Habitat-specific effects of food abundance on the condition of ovenbirds wintering in Jamaica. J. Anim. Ecol. 69: 883-895.

Swaddle, J. P. (1997) Within-individual changes in developmental stability affect flight performance. Behav. Ecol. 8: 601-604.

Swaddle, J. P. and Witter, M. S. (1997) On the ontogeny of developmental stability in a stabilized trait. Proc. R. Soc. Lond. B 264: 329-334.

Talloen, W., Lens, L., Van Dongen, S. and Matthysen, E. (in press) Feather development under environmental stress: lead exposure effects on growth patterns and realized growth. Bird Study.

Terrin, N., Schmid, C. H. and Lau, J. (2005) In an empirical evaluation of the funnel plot, researchers could not visually identify publication bias. J. Clin. Epidemiol. 58: 894-901.

Van Dongen, S. (1998) How repeatable is the estimation of developmental stability by fluctuating asymmetry? Proc. R. Soc. Lond. B 265: 1423-1427.

Van Dongen, S. (1999) Accuracy and power in fluctuating asymmetry studies: effects of sample size and number of within-subject repeats. J. Evol. Biol. 12: 547-550.

Van Dongen, S. (2007) What do we know about the heritability of developmental instability? Answers from a Bayesian model. Evolution 61: 1033-1042.

Van Dongen, S., Molenberghs, G. and Matthysen, E. (1999) The statistical analysis of fluctuating asymmetry: REML estimation of a mixed regression model. J. Evol. Biol. 12: 94-102.

Van Nuffel, A., Tuyttens, F. A. M., Van Dongen, S., Talloen, W., Van Poucke, E., Sonck, B. and Lens, L. (2007) Fluctuating asymmetry in broiler chickens: a decision protocol for trait selection in seven measuring methods. Poultry Science 86: 2555-2568.

Van Poucke, E., Van Nuffel, A., Van Dongen, S., Sonck, B., Lens, L. and Tuyttens, F. (in press) Experimental stress does not increase fluctuating asymmetry of broiler chickens at slaughter age Poultry Science.

Van Valen, L. (1962) A study of fluctuating asymmetry. Evolution 16: 125-142.

Velando, A. (2002) Experimental manipulation of maternal effort produces differential effects in sons and daughters: implications for adaptive sex ratios in the blue-footed booby. Behav. Ecol. 13: 443-449.

Vrijenhoek, R. C. and Lerman, S. (1982) Heterozygosity and developmental stability under sexual and asexual breeding systems. Evolution 36: 768-776.

Watson, P. J. and Thornhill, R. (1994) Fluctuating asymmetry and sexual selection. Trends Ecol. Evol. 9: 21-25.

Whitlock, M. (1996) The heritability of fluctuating asymmetry and the genetic control of developmental stability. Proc. R. Soc Lond. B 263: 849-854.

Whitlock, M. (1998) The repeatability of fluctuating asymmetry: a revision and extension. Proc. R. Soc Lond. B 265: 1428-1430.

Willis, K. J. and Whittaker, R. J. (2002) Ecology: species diversity-scale matters. Science 295: 1245-1248.

\section{LUC LENS*}

Terrestrial Ecology Unit, Department of Biology, Ghent University, K. L. Ledeganckstraat 35, B-gooo Ghent, Belgium.

\section{HILDE EGGERMONT}

Limnology Unit, Department of Biology, Ghent University, K. L. Ledeganckstraat 35, B-9ooo Ghent, Belgium.

*Author for correspondence; e-mail:Luc.Lens@UGent.be 